Boletín de la Sociedad Geológica Mexicana

VOLUMEN 65, NÚM. 1, 2013, P. 137-156

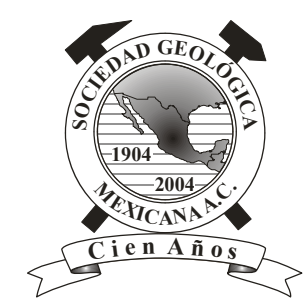

\title{
Geología y magnetometría aérea del Graben de Villa de Reyes, San Luis Potosí, Mesa Central de México: implicaciones tectónicas y geohidrológicas
}

\author{
Héctor López-Loera ${ }^{1, *}$, Margarito Tristán-González² \\ ${ }^{1}$ Instituto Potosino de Investigación Científica y Tecnológica A.C. Camino a la presa San José 2055, Lomas 4a sección C.P. 78216, \\ San Luis Potosí, S.L.P. \\ ${ }^{2}$ Instituto de Geología, Universidad Autónoma de San Luis Potosí; Av. Dr. Manuel Nava No. 5, Zona Universitaria, San Luis Potosí, \\ San Luis Potosí, México 78240. \\ *hlopezl@ipicyt. edu.mx
}

\begin{abstract}
Resumen
El estudio realizado está enfocado a conocer la geología y tectónica del subsuelo en el Graben de Villa de Reyes. Para ello se procesan datos aeromagnéticos por medio de una serie de filtros matemáticos que permiten resaltar información en unos casos u obscurecerla en otros, con el fin de observar y analizar estructuras geológicas superficiales o profundas. El estudio permitió identificar zonas de falla y/o fracturas bajo la cubierta aluvial, así como identificar zonas de fuente bajo los depósitos volcánicos en los hombros del Graben de Villa de Reyes. Los lineamientos aeromagnéticos interpretados permitieron identificar las zonas de recarga del acuífero de la zona.

Palabras clave: Graben de Villa de Reyes, magnetometría aérea, geología, fallas y/o fracturas, lineamientos magnéticos.

\section{Abstract}

The study is focused on learning about the underground geology and tectonics in the Villa de Reyes Graben. The aeromagnetic data is processed through a series of mathematical filters that allow us to increase the resolution of weak anomalies in some cases or, in others, to simplify the appearence of magnetic maps by suppressing local features in order to observe and analyze surface or deep geological structures. The study allowed us to identify fault and/or fractures zones that are under alluvial cover, and to identify sources of volcanic activity beneath the existing volcanic deposits on the shoulders of the Villa de Reyes Graben. The interpreted aeromagnetic trends allowed us to identify the aquifer recharge zone in the area.
\end{abstract}

Keywords: Villa de Reyes Graben, aeromagnetometry, geology, fault and/or fractures, magnetic trends. 


\section{Introducción}

Uno de los grandes problemas de México es asegurar el abasto de recursos hidráulicos a la población para uso humano e industrial, ya sea por medio de fuentes superficiales o por la perforación de pozos para la extracción de agua subterránea; desafortunadamente la falta de este líquido vital es cada día mayor y el estado de San Luis Potosí no es la excepción.

El estado de San Luis Potosí se ubica al norte del Altiplano Central localizado en la parte centro-oriental de la República Mexicana, entre los paralelos $21^{\circ} 09^{\prime} 35^{\prime \prime}$ y $24^{\circ} 33^{\prime} 09^{\prime \prime}$ de latitud norte y los meridianos $98^{\circ} 19^{\prime} 52^{\prime \prime}$ y $102^{\circ} 17^{\prime} 51^{\prime \prime}$ de longitud al oeste de Greenwich (Figura 1). El estado está dividido en cuatro regiones naturales: El Altiplano, el Centro, la Zona Media y La Huasteca, con un total de $62304.74 \mathrm{~km}^{2}$ de extensión en la que habitan del orden de 2410400 personas (INEGI, 2009).

El estado de San Luis Potosí se divide en 2 regiones hidrogeológicas muy contrastantes: la región del Salado localizada en la porción central y norte del estado y con una superficie dentro del mismo de $35164.19 \mathrm{~km}^{2}$, presenta un patrón hidrológico de tipo dendrítico endorreico donde las corrientes de agua superficiales son intermitentes, escasas y de poco caudal que sólo fluyen durante la época de lluvias en verano y ocasionalmente en invierno. La segunda es la región del Pánuco localizada en la porción sur y sureste del estado y con una superficie dentro del mismo de 27140.55 $\mathrm{km}^{2}$ y tiene una densa red fluvial de corrientes perennes que conforman algunos ríos que desembocan en el Golfo de México (CRM, 1992).

En la ciudad capital de San Luis Potosí uno de los problemas sociales relevantes es el de asegurar el abastecimiento de agua para fines humanos e industriales, ya que la demanda crece año con año y localmente el acuífero se encuentra sobre explotado, por lo que se considera necesario estudiar otras áreas del sistema acuífero regional de San Luis Potosí-Villa de Reyes, alejadas del cono de abatimiento de sobreexplotación de la mancha urbana de la ciudad de San Luis Potosí, en los últimos 30 años el nivel piezométrico ha descendido 80 metros en el centro y alcanzando en algunas zonas un descenso de hasta $3 \mathrm{~m}$ por año (Ramos-Leal et al., 2007).

El área estudiada se localiza en la parte central de México, en la provincia fisiográfica llamada Mesa Central, la cual se caracteriza por ser una zona semiárida en donde la principal fuente de abastecimiento de agua está constituida por agua subterránea y en ocasiones llega a ser la única fuente para satisfacer las necesidades de este elemental líquido.

Este estudio tiene como objetivo: conocer las posibilidades acuíferas que existen en las rocas del

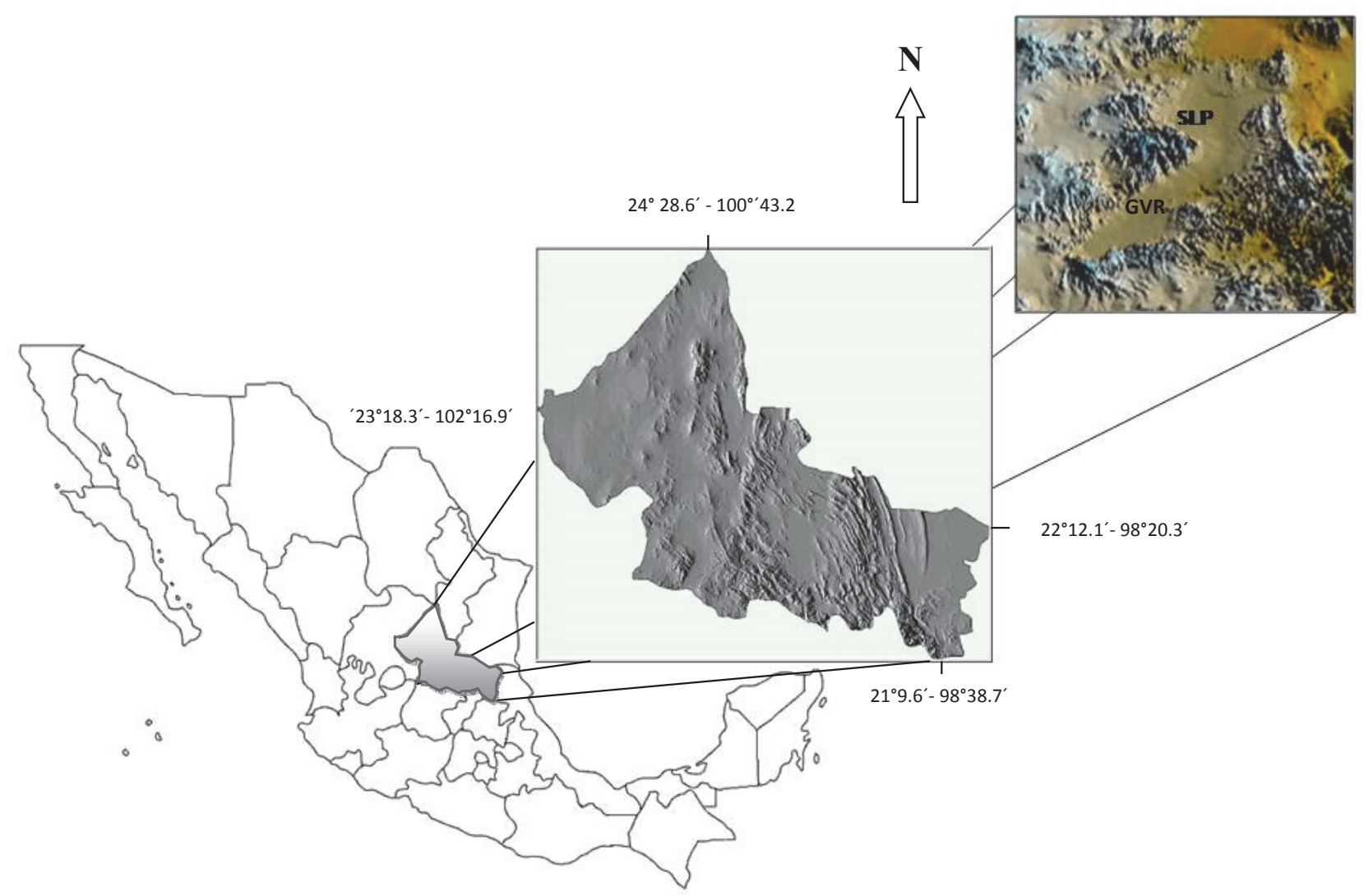

Figura 1. Mapa de localización del Graben de Villa de Reyes (GVR), Estado de San Luis Potosí, México. SLP=Cd. de San Luis Potosí. 
basamento rocoso del Graben de Villa de Reyes, así como la tectónica del subsuelo en base a estudios geológicos y geofísicos magnéticos.

La mayoría de los estudios exploratorios geofísicos para agua subterránea se realizan con métodos eléctricos y electromagnéticos, y rara vez se utilizan métodos de campo natural como lo es el método magnético. Debido a que las rocas volcánicas en su mayoría contienen en mayor o menor medida minerales ferromagnéticos, el estudio del magnetismo asociado a ellas permite la localización de zonas permeables que pueden contener agua. Los métodos magnéticos en aplicaciones geológicas iniciaron en los años de 1630 en Suecia, utilizándolos en la prospección de mineral de fierro (Hanna, 1990). El estudio de los métodos magnéticos a problemas geológicos ha ido avanzando en paralelo con el desarrollo de los magnetómetros. En la industria petrolera se ha utilizado como métodos de reconocimiento para estudios regionales desde principios del siglo pasado y con el desarrollo del magnetómetro aéreo en 1941 (Blakely, 1995) se facilitaron las mediciones magnéticas a lugares de difícil acceso por tierra, además, se empezó a cubrir mayor superficie en menor tiempo, esto es una gran ventaja de esta técnica exploratoria. A pesar de las múltiples ventajas de este método, poco uso se le ha dado en la prospección de agua subterránea, probablemente debido a que las propiedades magnéticas no se relacionan directamente con las propiedades que se asocian a la existencia de agua subterránea. Las técnicas magnéticas miden el campo magnético remanente asociado con cambios en el campo magnético de la Tierra asociada a la estructura geológica.

\section{Marco Geológico}

El Graben de Villa de Reyes (GVR) es una fosa tectónica de edad oligocénica (Tristán-González, 1986), limita en su porción septentrional a dos campos volcánicos, el Campo Volcánico de San Luis Potosí (CVSLP) y el Campo Volcánico del Río Santa María (CVRSM), y en su porción meridional involucra también al sur al Campo Volcánico de la Sierra de Guanajuato. Estos campos están formados por un paquete de rocas volcánicas que varían de dacitas a riolitas de alto sílice, eruptadas en el periodo comprendido entre los 32 y $28 \mathrm{Ma}$, donde el $80 \%$ es de carácter efusivo y el resto corresponde a productos piroclásticos asociados a la apertura de los conductos por donde posteriormente salieron las lavas (Labarthe-Hernández et al., 1982; Tristán-González, 1986). Esta fosa se encuentra rellena por depósitos volcánicos y clásticos de edad Oligoceno temprano al reciente. Su longitud es de aproximadamente $200 \mathrm{~km}$ con un ancho variable de 10-20 km, el relieve es en las partes más bajas de la porción norte del Valle de San Luis Potosí de unos $1800 \mathrm{~m}$, y en la parte SW en la zona de Nuevo Valle de Moreno, Guanajuato de 2230 m. El fondo de la fosa está conformado por domos exógenos de la Latita Portezuelo de 30.6 Ma (Labarthe-Hernández et al., 1982), y en algunos sitios llega a aflorar como picachos, como se observa en las inmediaciones del poblado de Villa de Reyes y en el Alto de la Melada al NE de la ciudad de San Luis Potosí. En los pozos se han cortado columnas de la Latita Portezuelo hasta de $500 \mathrm{~m}$ en las inmediaciones de Villa de Reyes (Labarthe-Hernández et al., 1982; AguirreHernández, 1992). La otra roca volcánica importante sepultada, son productos piroclásticos post-colapso, que corresponden a los dos miembros de la Ignimbrita Panalillo de edad $26 \mathrm{Ma}$ (Labarthe-Hernández et al., 1982). La Latita Portezuelo sobreyace en el fondo de la fosa a los sedimentos marinos del Cretácico tardío, a la Formación Cenicera y a la Ignimbrita Santa María. Los sedimentos marinos solo se han detectado por la perforación de pozos en dos sitios; en la localidad de Cándido Navarro al NE de la ciudad de San Luis Potosí, donde se cortó la Formación Indidura a $600 \mathrm{~m}$. El otro pozo se localiza en el poblado de Estanzuela, unos $15 \mathrm{~km}$ al NW de la ciudad de San Luis Potosí, cortando a la Formación Indidura a $451 \mathrm{~m}$ (Martínez-Ruiz y CuellarGonzález, 1979; Labarthe-Hernández et al., 1982; AguirreHernández, 1992).

El GVR forma parte de una serie de estructuras tectónicas presentes en la Mesa Central que corresponde a la etapa de máxima extensión del área de 26-28 Ma (Labarthe-Hernández et al., 1982; Tristán-González, 1986; Nieto-Samaniego et al., 1997; Henry y Aranda-Gómez, 1992; Aranda-Gómez et al., 2000) y que corresponde a la provincia de Cuencas y Sierras de la República Mexicana, la cual se ha considerado como prolongación hacia el sur de la Provincia "Basin and Range" (Stewart, 1998), que se extiende unos $1100 \mathrm{~km}$ desde el SW de los Estados Unidos hasta el límite norte de la Faja Volcánica Trans-Mexicana (Henry y Aranda-Gómez, 1992). Las principales estructuras de esta provincia son fosas y pilares tectónicos (fosas, semi-fosas y pilares tectónicos) que localmente forman bloques basculados (Stewart, 1998; Henry y ArandaGómez, 1992). El GVR en el Campo Volcánico de San Luis Potosí se encuentra cruzado por un sistema de fallas de orientación NW-SW, que se arreglan en un patrón en dominó, y en menor cantidad fosas tectónicas estrechas como la de Bledos, Enramadas, Paso Blanco y Santa Rosa, las cuales se rellenaron con productos piroclásticos de la Ignimbrita Panalillo y basaltos en una etapa de vulcanismo bimodal (Labarthe-Hernández y De la Huerta-Cobos, 1998; Rodríguez-Ríos, 2003; Torres-Aguilera, 2005), Figura 2.

\section{Estratigrafía}

La geología del área estudiada se puede separar en tres paquetes: el perteneciente al Complejo Volcánico de la Sierra de San Miguelito (CVSSM), el que se localiza dentro del GVR y el paquete del hombro SE, que forma parte del occidente del Campo Volcánico del Río Santa María (Figura 4). 


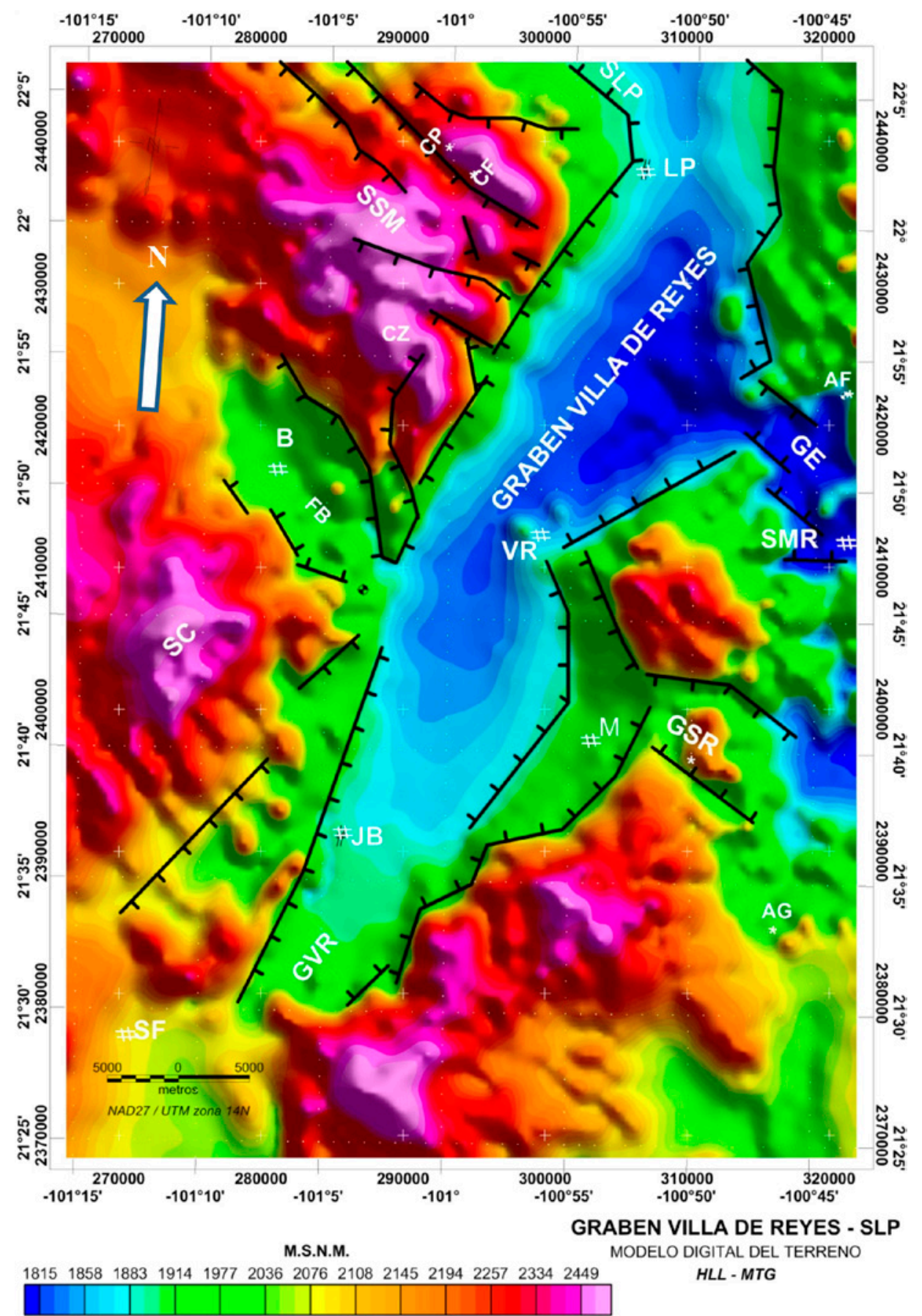

Figura 2. Modelo digital del terreno del Graben de Villa de Reyes, S.L.P., en donde se pueden observar algunas de las poblaciones y las principales estructuras tectónicas. $\mathrm{B}=$ Bledos: $\mathrm{JB}=$ Jaral de Berrios; $\mathrm{M}=$ Melchor; $\mathrm{LP}=\mathrm{La}$ Pila; $\mathrm{SF}=$ San Felipe; SLP $=$ San Luis Potosí; SMR $=$ Santa María del Río; VR = Villa de Reyes; SC = Sierra de Carranco; SMM = Sierra San Miguelito; GVR = Graben de Villa de Reyes; FB = Fosa de Bledos; GE = Graben Enramadas; GSR = Graben de Santa Rosa; CP = Cerro del Potosí; CF = Cerro del Fraile; CZ = Cerro Zapote; AF = Andesita Agua Fría; AG = Andesita Golondrinas.

3.1. Paquete del Complejo Volcánico de la Sierra de San Miguelito (CVSSM) y Graben de Villa de Reyes (GVR).

\subsubsection{Cretácico tardio marino}

Las rocas pre-volcánicas del CVSSM y GVR corresponden a facies marinas de tipo flysch del Cretácico tardío correspondientes a la Formación Caracol (LabartheHernández et al., 1982), aunque también se ha sugerido que correspondan a rocas pertenecientes al Terreno Guerrero por la presencia de algunos fósiles presentes en lentes de caliza (Aguillón-Robles et al., 2004), su litología predominante consta de una intercalación de capas de espesor delgado de areniscas de color amarillo ocre y lutita de color gris oscuro, 
y esporádicamente se intercalan algunas capas y lentes de caliza carbonosa de color oscuro. Estos afloramientos son ventanas en la margen NE del GVR, al nor-poniente del poblado de Villa de Reyes (Figura 3) y se han localizado en el fondo del norte GVR a una profundidad de $600 \mathrm{~m}$.

\section{a) Ignimbrita Santa María}

La secuencia sedimentaria anterior al CVSSM fue cubierta en el Oligoceno temprano por flujos piroclásticos voluminosos de la Ignimbrita Santa María (Aguillón-Robles et al., 2004; Tristán-González et al., 2009). Esta roca aflora como ventanas en la margen nor-poniente y en el fondo del GVR (Figura 3). Se trata de una roca rica en fenocristales de cuarzo y en menor cantidad sanidino y plagioclasa, en matriz parcialmente desvitrificada. Contiene pómez colapsada y la matriz está por lo general oxidada, tiene una estructura columnar típica de las ignimbritas, su base es un vitrófido de color negro lenticular. Su espesor varía entre 60-100 m.

\section{b) Latita Portezuelo}

Esta roca ocupa gran parte de ambos hombros de la porción nor-oriental del GVR (Figura 3), consiste de una roca de color gris-café de textura porfirítica con fenocristales de plagioclasa $>$ sanidino $>$ cuarzo, con abundante magnetita diseminada. La matriz está desvitrificada, se clasificó con análisis químicos de elementos mayores como una roca riodacítica y su edad K/Ar es de $31.0 \pm 0.7$ Ma (TristánGonzález et al., 2009). Estas lavas formaron cadenas largas de domos exógenos que se emplazaron a través de fallas de orientación N-NW. Gran parte de estos domos se encuentran sepultados debajo de un paquete grueso de productos volcánicos y clásticos dentro del GVR, donde forman una topografía muy irregular detectada por la perforación de pozos. Su espesor es mayor dentro de la fosa que en sus hombros y puede alcanzar los $600 \mathrm{~m}$. De acuerdo con los pozos que se han perforado dentro de la fosa, se ha detectado

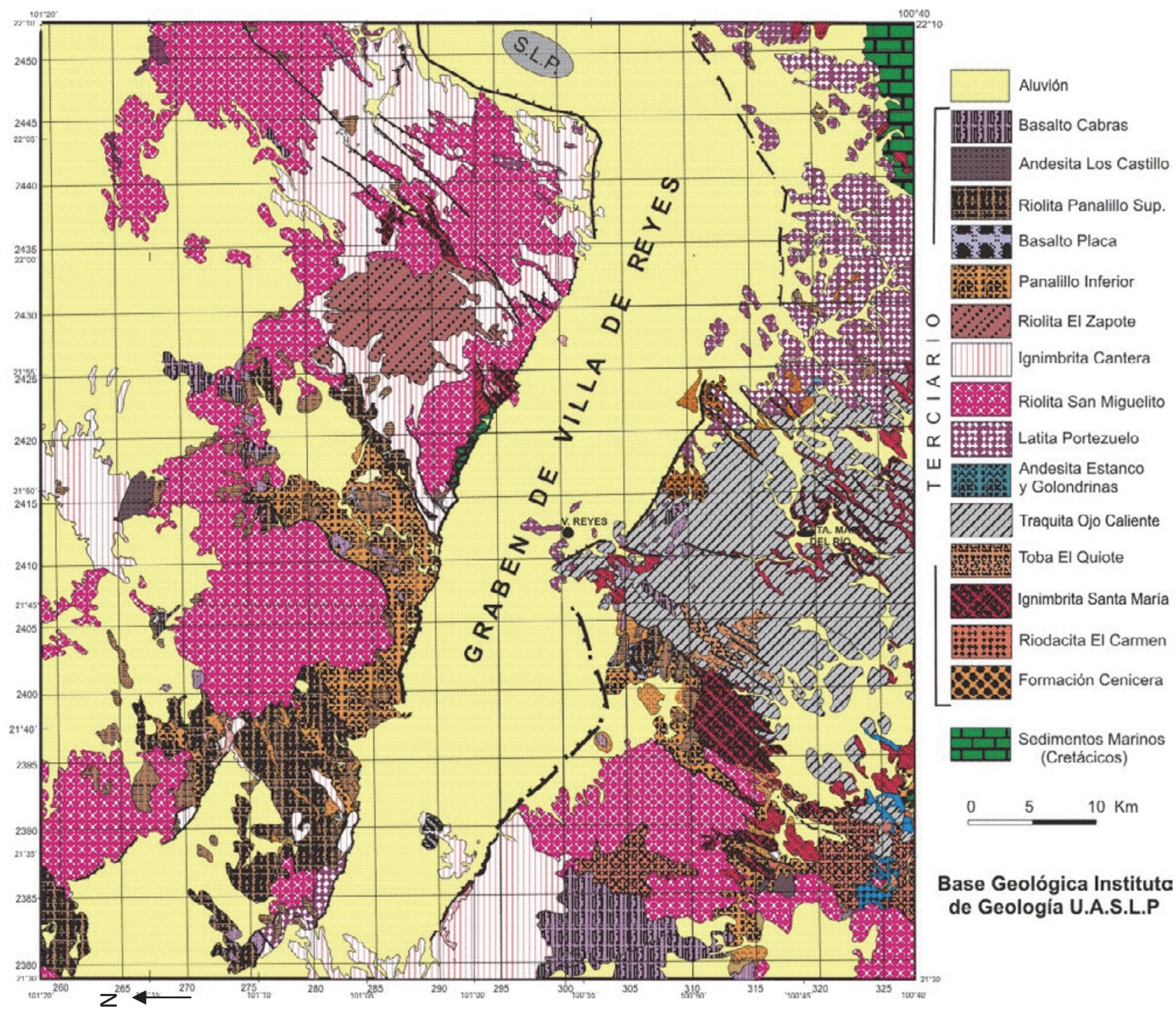

Figura 3. Mapa geológico del Graben de Villa de Reyes. (Modificado de Labarthe-Hernández et al., 1982). 


\section{S I M B O L O GÍ A}

\section{UATER NARIO}

\begin{tabular}{|l|}
\hline Qr \\
\hline Qal \\
\hline
\end{tabular}

Aluvión

Conglomerado Polimíctico

\section{T E R CIARIO}

\begin{tabular}{|c|c|}
\hline Cgps & Conglomerado Polimíctico Sup \\
\hline$T s c$ & Basalto Cabras \\
\hline Togpi & Conglomerado Polimíctico Inf \\
\hline Trp & Riolita Panalillo \\
\hline Tap & Ignimbrita Panalillo \\
\hline Trz & Riolita El Zapote \\
\hline Tic & Ignimbrita Cantera \\
\hline Tsm & Riolita San Miguelito \\
\hline lif: & Latita Portezuelo \\
\hline Ta & Andesita \\
\hline Toc & Traquita Ojo Caliente \\
\hline Tis & Ignimbrita Santa María \\
\hline$T c b$ & Andesita Casita Blanca \\
\hline $\operatorname{Tog}$ & Formación Cenicera \\
\hline
\end{tabular}

\section{CRETÁCICO}

\begin{tabular}{|c|c|}
\hline Ksc & Formación Caracol \\
\hline Kss & Formación Soyatal \\
\hline Ksi & Formación Indidura \\
\hline Kec & Formación Cuesta del Cura \\
\hline & Formación La Peña \\
\hline
\end{tabular}

ROCAS INTRUSIVAS

Intrusivo Rodrigo

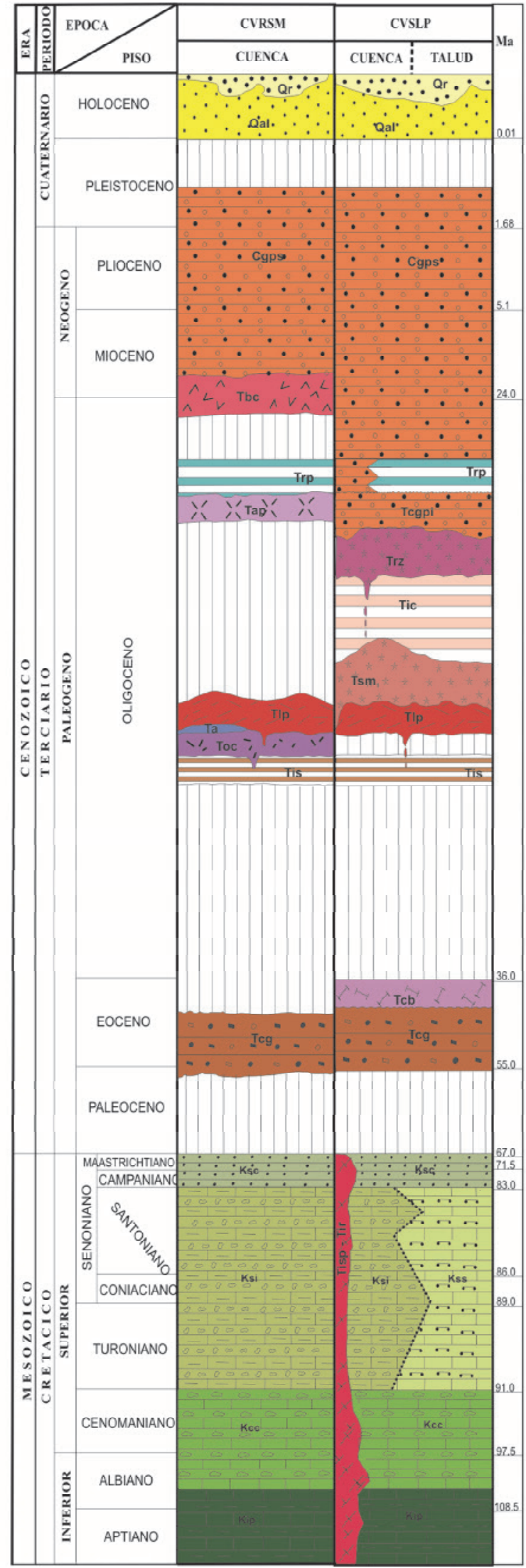

Figura 4. Columna estratigráfica de los Campos Volcánicos de San Luis Potosí (CVSLP) y del Río Santa María (CVRSM). (Modificada de Tristán González et al., 2008). 
que gran parte del acuífero profundo de agua termal se encuentra en fracturas de esta roca.

c) Riolita San Miguelito

La Riolita San Miguelito ocupa el mayor volumen de roca del CVSSM formando cuando menos cuatro domos gigantes: cerros Grande, Del Potosí- Fraile, Sierra de Carranco y Sierra las Mesas en la porción sur-este del GVR (Figura 3). Esta riolita está compuesta por varios pulsos de coladas de lava riolítica fluidal (Labarthe-Hernández et al., 1982). La mineralogía más común es la de una roca de color gris claro de textura porfirítica, con fenocristales de cuarzo $>>$ sanidino, en una matriz desvitrificada muy fluidal, con desarrollo de gran número de pliegues de flujo. Las lavas formaron domos gigantes y amplias coladas que se derramaron formando lóbulos hacia sus márgenes. Los domos se encuentran alineados en dirección NW-SE paralelos al conjunto de fallas que se formaron después de su salida. En su última fase eruptiva las coladas formaron un caparazón grueso en la cima de los domos, compuesto de brechas, con fragmentos de vidrio y roca desvitrificada, depósitos de calcedonia y óxidos de fierro, y formación de franjas de esferulitas por el enfriamiento rápido en superficie. Su espesor es variable debido a que son domos y por lo tanto el espesor es mayor en el centro, pudiendo alcanzar unos $800 \mathrm{~m}$ en el centro del CVSSM. La edad determinada por K/Ar fue de $30.0 \pm 1.5 \mathrm{Ma}$ (LabartheHernández et al., 1982). Estos domos son los generadores de estaño y topacio fumarólico en el área de la Sierra de San Miguelito y domos aledaños. Esta unidad no se localiza dentro del GVR.

\section{d) Ignimbrita Cantera}

Sobre la Riolita San Miguelito descansa un paquete de cuatro derrames piroclásticos que van de bien soldados hasta sin soldar. A este paquete se le asignó el nombre de Ignimbrita Cantera que aflora en gran parte de la Sierra de San Miguelito, sus espesores son variables pero puede alcanzar en el centro del CVSSM unos $300 \mathrm{~m}$. Por lo general es una roca de color café-rosáceo, de textura porfirítica. $\mathrm{Su}$ composición mineralógica es de fenocristales de cuarzo >> sanidino $>$ biotita, con pómez abundante y un $5 \%$ de líticos de color café en una matriz parcialmente desvitrificada. Los derrames con soldamiento alto poseen estructura columnar. Su composición química y mineralógica clasifica a este paquete ignimbrítico como de composición riolita. Su edad fue obtenida por K/Ar de $29.0 \pm 1.5 \mathrm{Ma}$ (Labarthe-Hernández et al., 1982). La Ignimbrita Cantera no se encuentra sepultada en el centro de la fosa, solo se ha detectado por pozos en los primeros escalones de las márgenes del GVR.

\section{e) Andesita Calderón}

En la intersección el GVR y el Graben de Bledos, aflora sobre la Ignimbrita Cantera, una roca de composición andesítica de distribución restringida a este sitio, que se le nombró como Andesita Calderón (Labarthe-Hernández et al., 1982). Se trata de una roca de textura porfirítica con fenocristales de plagioclasa y sanidino y como minerales accesorios zircón y apatito, en matriz parcialmente desvitrificada. Su espesor es de máximo $60 \mathrm{~m}$. Químicamente se le clasificó como una andesita rica en K. Su afloramiento está asociado a la margen del GVR.

\section{f) Riolita El Zapote}

En la porción más elevada de la Sierra de San Miguelito, sobre la Ignimbrita Cantera, se emplazaron los derrames de lava de la Riolita el Zapote (Labarthe-Hernández et al., 1982). Se trata de una roca de color gris claro de textura porfirítica, con fenocristales de cuarzo $\geq$ sanidino en una matriz desvitrificada y fluidal. Su espesor en la zona del Arroyo La Laja es de unos $130 \mathrm{~m}$, y de acuerdo con los análisis químicos de elementos mayores se le clasificó como una Riolita rica en K; su edad fue determinada de $29.2 \pm$ 0.8 (Aguillón-Robles et al., 1994). Estos derrames de lava formaron un domo amplio en lo más alto de la Sierra de San Miguelito (Figura 3). Otros domos más pequeños se encuentran al poniente del CVSSM y se caracterizan por tener topacio y estaño fumarólico.

\section{g) Riolitas Panalillo}

Contemporáneo al periodo de máxima extensión (28-26 $\mathrm{Ma}$ ) donde se formaron las principales fosas tectónicas y sistema de fallas normales NW-SE del CVSLP, estas fallas sirvieron de conducto para nuevas erupciones piroclásticas sintectónicas con el nombre de Riolita Panalillo Inferior y Superior (Labarthe-Hernández et al., 1982). El paquete inferior de la Riolita Panalillo es una secuencia de oleadas piroclásticas, depósitos de flujo de caída y flujos sin soldar. Los componentes de estos flujos de ceniza son por lo general ricos en líticos y pómez sin colapsar. Su composición mineralógica es de un $5 \%$ de fenocristales de cuarzo > sanidino $>$ biotita. Las oleadas y depósitos de caída se intercalan en los diferentes niveles de los flujos. Estos flujos rellenaron principalmente las fosas, aunque fuera de ellas hay remanentes que se acumularon rellenando depresiones. Su espesor dentro del GVR detectado por el corte de pozos es muy irregular, variando de $40-150 \mathrm{~m}$.

El miembro Superior de la riolita Panalillo es una roca de color gris-café de textura porfirítica, eutaxítica, con un 5-8 \% fenocristales de cuarzo > sanidino en una matriz parcialmente desvitrificada y bien soldada. Su base es un vitrofido lenticular que no sobrepasa el metro, pasando este a una zona esferulítica producto de la desvitrificación y en su cima una zona con abundante litofísas rellenas de calcedonia. Su espesor no es mayor a los $20 \mathrm{~m}$ y su edad es de $26.8 \pm 1.3 \mathrm{Ma}$ (Labarthe-Hernández et al., 1982). Dentro del GVR esta roca se encuentra sepultada por depósitos lacustres y clásticos, y su espesor detectado en la mayoría de los pozos varía entre 20-50 $\mathrm{m}$ y en los hombros del GVR no sobrepasa los $20 \mathrm{~m}$ y por lo general está asociado a las fallas principales. En algunos sitios, principalmente dentro 
de la Fosa de Bledos entre los dos miembros de la riolita Panalillo se encuentran coladas de basalto (Basalto La Placa) que no superan los $20 \mathrm{~m}$ de espesor. Su mineralogía consiste de fenocristales de andesina y olivino alterado. Este evento contemporáneo a los depósitos de la Riolita Panalillo se le considera bimodal (Torres-Aguilera y Rodríguez-Ríos, 2005).

h) Andesita Los Castillo

En la porción sur-occidental del CVSSM al sur-oriente del valle de Villa de Arriaga, se encuentran afloramientos en un área de $10 \mathrm{~km}^{2}$, de una roca de color café a gris oscuro, de textura porfirítica con 5-10\% de fenocristales de plagioclasa en una matriz formada de microlitos de plagioclasa con abundante magnetita oxidada. Se clasificó con elementos mayores quedando como una traquita. Su espesor no supera los $50 \mathrm{~m}$. Su edad fue calculada con K/Ar dando $20.5 \pm 0.5$ Ma (Tristán-González et al., 2009).

\section{i) Basalto Cabras}

En la porción NW del Graben de Bledos se encuentran remanentes de coladas basálticas pertenecientes al Basalto Cabras, que se describió como una roca de color negro de textura microporfirítica, los microfenocristales son de andesina y olivino alterado, con matriz de microlitos de plagioclasa. Su edad fue determinada por K/Ar de $21.5 \pm$ $0.3 \mathrm{Ma}$ (Tristán-González et al., 2009). Hacia la porción del hombro sur-poniente del GVR también hay remanentes esporádicos de esta roca.

\subsection{Secuencia del Campo Volcánico del Río Santa María, CVRSM}

El occidente del CVRSM y el CVSSM tienen en común el basamento de rocas marinas cretácicas de la Formación Caracol, Ignimbrita Santa María, Basalto Cabras y Riolita Panalillo Inferior ya descritas, por lo que, aquí ya no se describirán.

\subsubsection{Formación Cenicera}

Este depósito aflora en una ventana en la inmediación NE del poblado de Villa de Reyes en el Arroyo de La Cenicera, donde se describió como una secuencia de conglomerado bien consolidado, con fragmentos de caliza, arenisca, pedernal y algunos de rocas volcánicas, soportados en una matriz de arena. También presenta horizontes de limolita abundante hacia su cima. Este depósito fue nombrado como Conglomerado Cenicera (Labarthe-Hernández et al., 1982), esta unidad se correlaciona con los Conglomerados Rojos (Edwards, 1956). Su edad de acuerdo a trabajos realizados en el norte del CVSLP se basó en la determinación radiométrica de una muestra de andesita intercalada entre ellos, dando una edad K/Ar de $44.1 \pm 2.2 \mathrm{Ma}$, lo cual sitúa a este depósito en el Eoceno (Labarthe-Hernández et al., 1982). Este depósito fue cortado en el GVR en un pozo localizado al NE de la Ciudad de San Luis Potosí a los 528 m (Aguirre-Hernández, 1992). En el área del Arroyo La Cenicera subyace a la Ignimbrita Santa María.

\subsubsection{Riodacita del Carmen}

Es una roca de color café rojizo o amarillento, de textura porfirítica con un $15 \%$ de fenocristales hasta de $6 \mathrm{~cm}$ de plagioclasa, sanidino, cuarzo y óxidos de fierro con matriz desvitrificada. En algunos sitios se encuentra muy alterada, con nódulos de óxidos de fierro. En la zona de Tierra Nueva a esta roca se le conoce como Sangre de Pichón, donde presenta lajeamiento abundante, su extensión abarca prácticamente todo el CVRSM (Labarthe-Hernández et al., 1982). Su espesor en la zona de la población del Carmen es de unos $550 \mathrm{~m}$. Aflora como ventanas en el hombro suroriente del área del GVR (Figura 3).

\subsubsection{Andesita Estanco y Golondrinas}

Los principales afloramientos de la andesita Golondrinas se encuentran unos $15 \mathrm{~km}$ al sur de Santa María del Río, en las inmediaciones de la hacienda El Carmen (Figura 3). En ambos sitios la andesita se encuentra sobre la ignimbrita Santa María. Se trata de una roca de color negro, afanítica, con pequeños fenocristales muy aislados de plagioclasa y biotita, en una matriz compuesta de vidrio. El espesor es variable en el rango de 20-60 m (Labarthe-Hernández et al., 1982; Labarthe-Hernández et al., 1984).

\subsubsection{Toba El Quiote}

En la porción sur-oriental del GVR al SE de la población de Villa de Reyes se localizan ventanas de un flujo piroclastico sin soldar en su base y soldado en su cima, la roca es de color crema a rosa, con un $15 \%$ de fenocristales de cuarzo y sanidino, con pómez abundante y líticos. Su espesor en el Cerro del Quiote es de unos 50 m (LabartheHernández et al., 1983).

\subsubsection{Traquita Ojo Caliente}

La Traquita Ojo Caliente es una roca de color café, de textura porfirítica, con fenocristales de plagioclasa, sanidino y algunos clinopiroxenos alterados, en una matriz desvitrificada y fluidal, presenta varios derrames de lava con composición muy semejante. Hay zonas de brechas que corresponden principalmente a sus zonas de fuente. Estas lavas se encuentran formando cadenas de domos orientadas NW-SE. Los domos se encuentran afectados por fallas normales del mismo rumbo (NW-SE). Con análisis químicos de elementos mayores se clasificó a esta roca como una traquita rica en $\mathrm{K}$ (Labarthe-Hernández et al., 1982). Su edad determinada por K/Ar fue de $31.8 \pm 0.7$ Ma (Tristán-González et al., 2009). En la porción norte de esta zona del CVRSM, la Traquita Ojo Caliente subyace a la Latita Portezuelo del CVSLP. 


\section{Estudio Aeromagnético}

El levantamiento aeromagnético del área de estudio fue realizado por el entonces Consejo de Recursos Minerales (CRM, hoy Servicio Geológico Mexicano, SGM) en el año 1996, utilizando un avión Islander BN2-B27, equipado con un magnetómetro fijo de vapor de Cesio marca Scintrex CS-2 con una resolución de $0.001 \mathrm{nT}$ (nanoteslas), El vuelo realizado fue de contorno con una altura de vuelo de $450 \mathrm{~m}$ y con líneas de rumbo N-S a cada $1000 \mathrm{~m}$. en una dirección N-S. El sistema de adquisición de datos fue PDAS 1000 y para la edición de la información utilizaron video JVC de alta resolución.

El estudio aeromagnético aquí reportado consistió en el procesamiento, análisis e interpretación de datos digitales que se obtuvieron del SGM cubriendo una superficie de $4353 \mathrm{~km}^{2}$ (54.3 km N-S y $80.2 \mathrm{~km} \mathrm{E-W).} \mathrm{Los} \mathrm{datos} \mathrm{iníciales}$ fueron pre-procesados por el CRM (Bhattacharyya, 1971) y nosotros manipulamos la información digital a través de un software de la empresa Geosoft (Oasis-montaj Assembly Data Processing and Analysis System).

De la investigación magnética aérea se generó un mapa de la configuración del campo magnético residual (CMR, Figura 5) a través de sustraerle a los datos del campo magnético total el Campo de Referencia Geomagnético Internacional (IGRF) de 1995. También se realizaron mapas del CMR reducido al polo (CMRP) y este proceso se tomó como base para generar mapas de continuaciones analíticas a $1,2,3,5,7.5$ y $10 \mathrm{~km}$, primeras y segundas derivadas y señales analíticas.

Una de las ventajas de trabajar con el campo magnético reducido al polo (Figura 6) en lugar del campo magnético residual o total, es que las anomalías magnéticas se sitúan sobre las fuentes que las causan. Se hace el símil a través de un filtro matemático (Baranov y Naudy, 1964) de trasladar nuestra zona de trabajo al polo norte en donde la inclinación del campo magnético es vertical.

\subsection{Propiedades Magnéticas}

Los estudios de magnetometría aprovechan una de las rocas con mayor presencia en el GVR, la Latita Portezuelo porque tiene un mineral accesorio que es la magnetita, la cual le da una propiedad totalmente contrastante en cuanto a la susceptibilidad magnética respecto de las otras unidades geológicas existentes en el GVR y que permite relativamente su fácil reconocimiento en el subsuelo. La susceptibilidad magnética $(k)$ es el parámetro fundamental de la prospección geofísica magnética, dado que la respuesta de las rocas y minerales está determinada por la cantidad de material magnético según se desprende de la siguiente relación:

$$
I=k H \quad \text { (Ecuación 1) }
$$

En donde $I=$ Intensidad de magnetización; $k=$ Susceptibilidad magnética y $H=$ Fuerza del campo magnético.
Esta simple relación (Ecuación 1), nos establece que para una misma zona la fuerza del campo magnético $(H)$ es el misma, y si los valores de la intensidad de magnetización $(I)$, medida a través de un magnetómetro son diferentes, será porque la susceptibilidad magnética $(k)$ es diferente. La $k$ de las rocas tiene una rango de variabilidad que va desde $10^{-8}$ a 1 unidades S.I., lo cual nos permite caracterizar diferentes unidades geológicas (López-Loera y UrrutiaFucugauchi, 1999), Para esta zona de estudio se midieron $k^{\prime}$ s in-situ, en todas las unidades geológicas aflorantes en el área (Tabla 1), utilizando un susceptibilimetro portátil digital Exploranium-Kappameter KT-9 con una máxima sensibilidad de $1 \times 10^{-5}$ unidades S.I.

\subsection{Interpretación de Anomalías Magnéticas}

Con el fin de conocer las zonas de debilidad asociadas a fallas y/o fracturas que se asocian a zonas de recarga del acuífero del GVR se realizó un análisis de los lineamientos aeromagnéticos sobre la configuración del campo magnético reducido al polo por medio de diagramas de frecuencia y de peso (Figura 7).

Los resultados del análisis establecieron que los lineamientos aeromagnéticos presentan una dirección preferencial de $40^{\circ} \mathrm{NW}$ que es congruente con los rumbos generales de las estructuras geológicas en el Altiplano Mexicano.

Para la interpretación de las anomalías aeromagnéticas se utilizaron algunos filtros matemáticos en el procesamiento de la información, uno de ellos es el conocido como continuación ascendente del campo magnético (Henderson, 1970), que se utiliza para reducir los efectos de fuentes superficiales. Al cambiar el nivel de observación la respuesta disminuye, ya que la fuerza del campo magnético es inversamente proporcional al cuadrado de la distancia entre la fuente y el nivel de observación y por lo tanto la señal magnética se suaviza, se puede decir que el algoritmo de continuación ascendente es un filtro suavizador (Telford et al., 1990).

En el mapa de configuración de la continuación ascendente $2 \mathrm{~km}$ (Figura 8), se observa que el $\mathrm{N}$ de la Sierra de San Miguelito (SSM) está asociada con una anomalía positiva con intensidades de magnetización entre 5 y 10 nT que esta además limitada tanto al $\mathrm{N}$ como al $\mathrm{S}$ por lineamientos aeromagnéticos que se ven orientados en bloques paralelos al patrón principal de fallamiento normal. En el centro de la SSM se observa una anomalía de características negativas en forma alargada con valores de la intensidad del campo magnético del orden de los -20 a -30 nT que coincide con los afloramientos de la Riolita Zapote. Hacia el sur se tiene que el relleno piroclástico de la fosa de Bledos también está asociada a una anomalía negativa del orden de -20 nT con rumbo NW-SE, y que representa una porción de un lineamiento magnético regional de dirección N-S, caracterizado por bajos magnéticos que continúan hasta Jaral de Berrios, con anomalías asociadas a intensidades de 

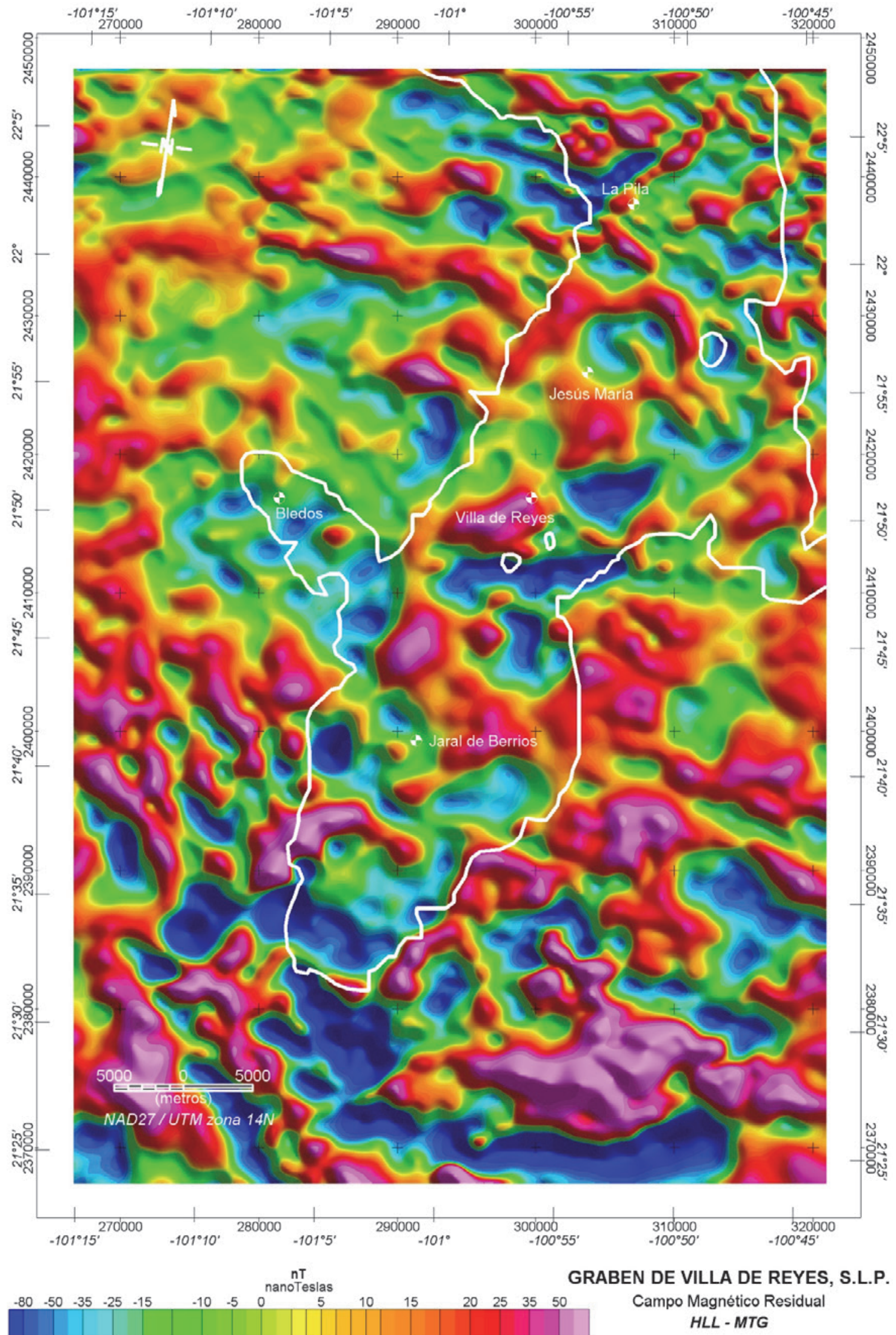

Figura 5. Mapa que muestra la configuración de la anomalía aeromagnética residual con el campo de georeferencia removido (IGRF). Se indica el límite del Graben de Villa de Reyes en línea color blanco. En el mapa se pueden observar la ubicación de algunas poblaciones como Villa de Reyes, Jaral de Berrios, Bledos, Jesús María y La Pila. 


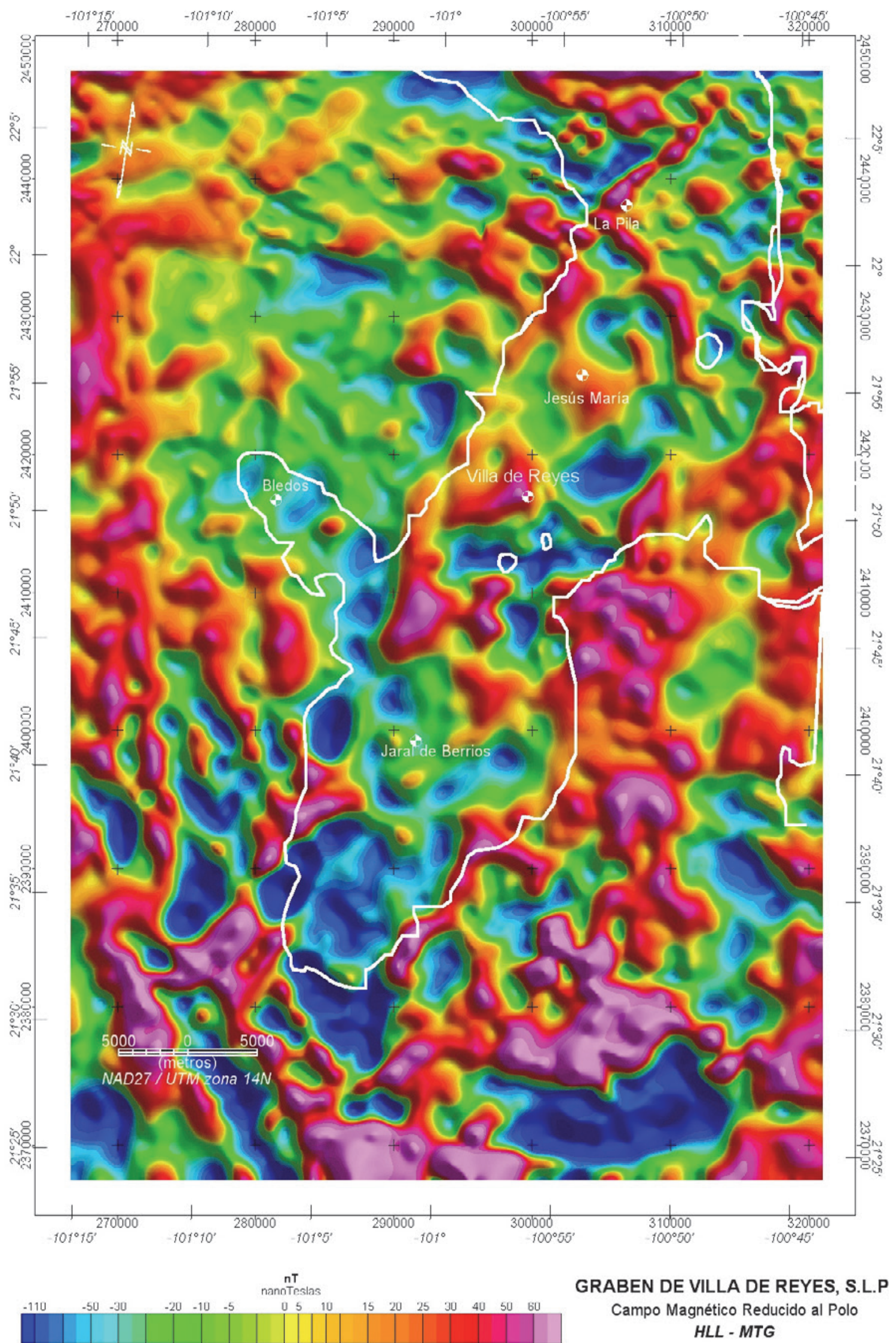

Figura 6. Mapa que muestra la configuración del campo magnético reducido al polo. Las anomalías magnéticas (máximos) que se observan dentro del Graben de Villa de Reyes están asociadas a la presencia de rocas volcánicas bajo la cubierta aluvial (mínimos). 
Tabla 1. Susceptibilidades magnéticas $(k)$ de las rocas de los hombros del Graben de Villa de Reyes, S.L.P.

\begin{tabular}{|c|c|c|c|c|c|c|}
\hline \multirow{2}{*}{ TIPO DE ROCA } & \multicolumn{2}{|c|}{ COORDENADAS } & \multicolumn{4}{|c|}{ SUSCEPTIBILIDAD MAGNÉTICA x $10^{-6}$ S.I. } \\
\hline & $\mathrm{X}$ & $\mathrm{Y}$ & No. Lecturas & Mínimo & Máximo & Promedio \\
\hline Tsm Intermedia & 300591 & 2431054 & 10 & 0.52 & 1.65 & 1.326 \\
\hline Tsm Alta & 300576 & 2431082 & 11 & 0.6 & 2.05 & 1.305 \\
\hline Tsm más Alta & 300564 & 2431109 & 14 & 0.32 & 8.47 & 3.732 \\
\hline Tlp Inferior & 301413 & 2414367 & 10 & 0.21 & 0.48 & 0.342 \\
\hline Tlp Medio & 301467 & 2414347 & 13 & 0.13 & 0.29 & 0.207 \\
\hline Tlp Superior & 301456 & 2414325 & 10 & 0.15 & 0.26 & 0.184 \\
\hline Tlp Inferior en VR & 298662 & 2412497 & 9 & 1.62 & 2.82 & 2.142 \\
\hline Tlp Intermedio VR & 298644 & 2412653 & 9 & 2 & 10.07 & 6.498 \\
\hline Tlp Superior en VR & 298653 & 2412610 & 13 & 1.84 & 12.01 & 5.516 \\
\hline Tlp vía del tren & 298674 & 2412512 & 88 & 0.4 & 9.82 & 3.331 \\
\hline $\mathrm{Cg}$ & 298674 & 2412512 & 8 & 0.69 & 9.65 & 2.208 \\
\hline Tlp Baja & 297376 & 2413049 & 20 & 0.35 & 1.97 & 1.084 \\
\hline Tlp Alta & 297376 & 2413049 & 10 & 0.96 & 1.73 & 1.333 \\
\hline Bitrofído Panalillo & 284231 & 2415531 & 12 & 0.92 & 1.32 & 1.108 \\
\hline Panalillo Superior & 284177 & 2415736 & 12 & 2.82 & 5.67 & 4.075 \\
\hline Basalto & 283903 & 2415750 & 20 & 0.74 & 2.38 & 1.527 \\
\hline Basalto Placa & 283406 & 2415601 & 18 & 0.65 & 8.24 & 1.911 \\
\hline Tap & 283262 & 2415182 & 21 & -0.07 & 0.35 & 0.08 \\
\hline Basalto & 284379 & 2413333 & 23 & 1.02 & 7.45 & 2.608 \\
\hline Tap & 284665 & 2412554 & 24 & 0.33 & 0.91 & 0.746 \\
\hline Trp Ventilla & 289352 & 2409281 & 28 & 1.45 & 6.65 & 3.332 \\
\hline Intr. Nuevo Valle & 289345 & 2409262 & 15 & 1.3 & 11.5 & 4.814 \\
\hline Tap Ventilla & 290045 & 2409239 & 13 & 0 & 0.1 & 0.033 \\
\hline Toc & 303840 & 2411295 & 11 & 0.58 & 1.63 & 1.056 \\
\hline Toc & 303825 & 2411277 & 10 & 0.95 & 2.12 & 1.462 \\
\hline Tis & 306638 & 2409928 & 17 & 0 & 1.1 & 0.437 \\
\hline Tbc rodados & 306632 & 2409912 & 12 & 3.65 & 9.49 & 6.639 \\
\hline Tic & 302973 & 2437443 & 10 & 1.1 & 1.66 & 1.324 \\
\hline Tlp & 302657 & 2437568 & 22 & 0.03 & 15.9 & 2.54 \\
\hline Tlp unidad 2 & 302625 & 2437777 & 11 & 5.48 & 15.8 & 10.06 \\
\hline Tic & 304341 & 2434057 & 20 & 0.81 & 1.59 & 1.22 \\
\hline Tic Zona Falla & 301160 & 2432183 & 10 & -0.16 & 0.06 & -0.069 \\
\hline Tic base & 301118 & 2432213 & 22 & 0.56 & 1.43 & 0.958 \\
\hline Dique Riolítico & 301133 & 2432246 & 10 & -0.05 & 0.22 & 0.014 \\
\hline Tis inferior & 300660 & 2430826 & 8 & 0.11 & 0.65 & 0.437 \\
\hline
\end{tabular}

Tsm = Riolita San Miguelito; Tlp = Latita Portezuelo; Tap = Riolita Panalillo Inferior; Trp = Riolita Panalillo Superior; Toc = Traquita Ojo Caliente; Tis = Ignimbrita Santa María; Tbs = Basalto Cabras; Tic = Ignimbrita Cantera; $\mathrm{Cg}=$ Conglomerado; S.I. = Sistema Internacional.

hasta $>-50 \mathrm{nT}$ y que cubren prácticamente todo el relleno del graben hacia el sur, con dimensiones aproximadas de $11 \mathrm{~km}$ en dirección E-W y de $17 \mathrm{~km}$ en dirección N-S.

Hacia el norte del mapa se observa una anomalía magnética negativa con intensidades del orden de los -20 a $-30 \mathrm{nT}$ que se correlaciona con la zona de falla SW al S de la ciudad de San Luis Potosí, donde la respuesta magnética se debe a la presencia de la Ignimbrita Cantera $(k=-0.069$ x $10^{-6}$ a 10.06 x $10^{-6}$ S.I.). En la zona del Arroyo El Meco en donde la fosa sufre una inflexión hacia el norte, se refleja una anomalía de 0 a $7 \mathrm{nT}$, que deben corresponder a los altos de la Latita Potezuelo ( $k=0.03 \times 10^{-6}$ a $6.29 \times 10^{-6}$ S.I. $)$ que se encuentra debajo de la Riolita San Miguelito $\left(k=1.3 \times 10^{-6}\right.$ a 3.7 × $10^{-6}$ S.I. ) e Ignimbrita Cantera. Esta anomalía se debe a la presencia de la abundancia de magnetita diseminada que contiene en su matriz la latita.

Hacia el interior del GVR, en la cubierta aluvial y hacia el SW y NW de la población del mismo nombre se identifica una franja (trend) de anomalías magnéticas positivas con valores de la intensidad del campo magnético del orden de 10 a $20 \mathrm{nT}$, que hacia el SW tiene una dirección NW-SE y hacia el NW cambia a NE-SW y que se han asociado como a altos de los domos de la Latita Portezuelo en el subsuelo de la fosa y quizá donde los domos tienen mayor espesor. La Comisión Federal de Electricidad (CFE, 1990) reportó espesores de la latita Portezuelo hasta de $500 \mathrm{~m}$.

En la configuración del campo magnético reducido al polo y continuado ascendentemente $2 \mathrm{~km}$ se puede observar 
Diagrama de frecuencias de los lineamientos aeromagnéticos en el graben de Villa de Reyes.
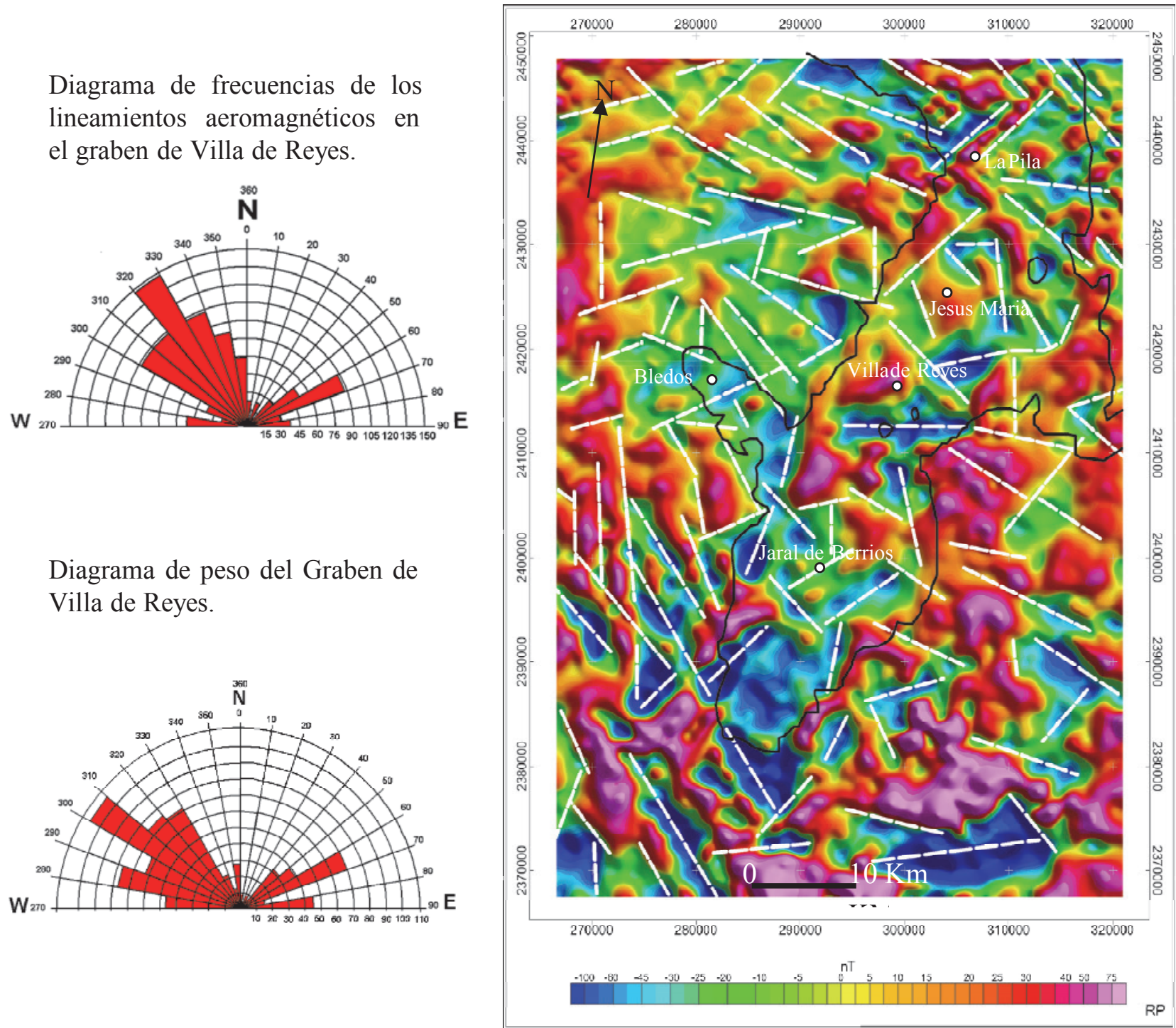

Diagrama de peso del Graben de Villa de Reyes.

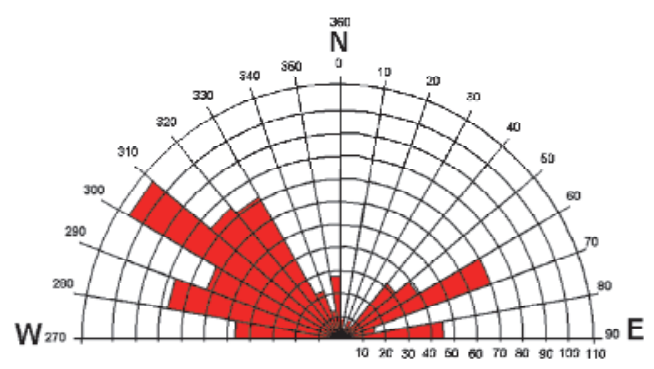

interpretación de la información es la derivada vertical del

que en gran parte del hombro SE del GVR existe una serie de anomalías positivas que constituyen un trend magnético de dirección casi N-S, con valores de la intensidad del campo magnético del orden de los 15 a los $30 \mathrm{nT}$, que deben corresponder a la franja del basalto Cabras $(k=3.65$ $\times 10^{-6}$ a $9.49 \times 10^{-6}$ S.I. ) que se encuentra descansado en las partes altas de las rocas riolíticas. Los altos magnéticos con valores del orden de los $30 \mathrm{nT}$, de este trend magnético deben de corresponder a las zonas fuente por donde salieron los basaltos y el resto a sus coladas. Paralelo a la traza de la falla principal del GVR se aprecian anomalías positivas con valores de la intensidad magnética del orden de 5 a $15 \mathrm{nT}$ y que son la respuesta a los afloramientos principalmente de los domos de la Latita Portezuelo y Traquita Ojo Caliente $\left(k=0.73 \times 10^{-6} \mathrm{a} 2.12 \times 10^{-6}\right.$ S.I. $)$.

Otro de los filtros utilizados en el procesamiento e campo magnético (Henderson y Zietz, 1949) que se utilizan principalmente para resaltar contactos y pequeños rasgos asociados con anomalías magnéticas, se dice que la primera derivada es el gradiente vertical (Robinson y Coruh, 1988; Ecuación 2), ya que nos informa sobre la razón del cambio de la anomalía con la altura.

$$
\left(T_{z+\Delta z}-T_{z}\right) / \Delta z=\Delta T / \Delta z=T^{\prime} \quad \text { (Ecuación 2) }
$$

Donde $T^{\prime}$ es la primera derivada y $z=$ altura; $T$ intensidad magnética total;

En mapa de la configuración de la primera derivada vertical $(1 \mathrm{aDz})$ del campo magnético reducido al polo, nos muestra como las anomalías tienden a agudizarse en los bordes y mejorar sus características superficiales. Se observa claramente la correlación que existe entre los límites del GVR y los lineamientos magnéticos. Para cuestión de 

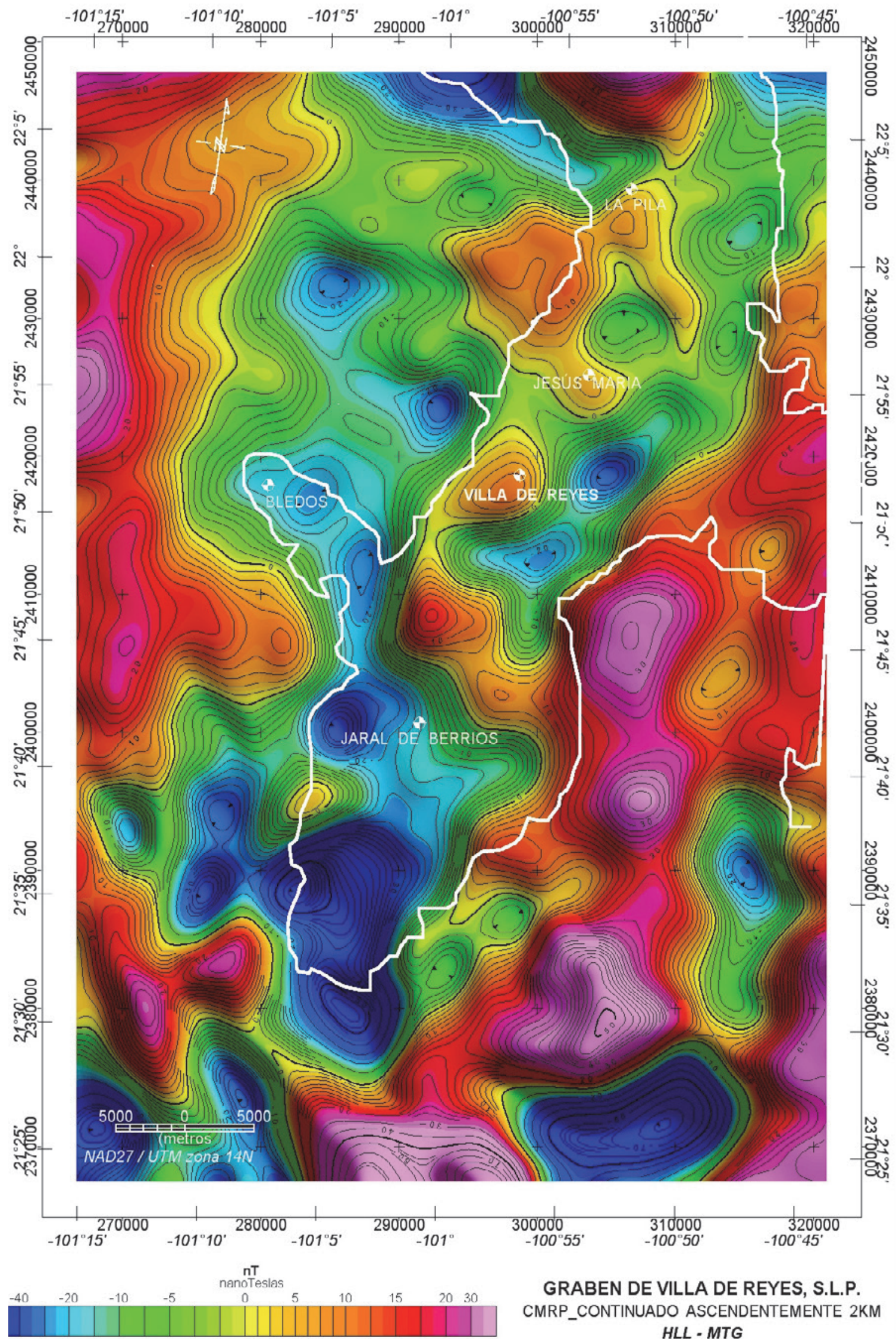

GRABEN DE VILLA DE REYES, S.L.P.

CMRP_CONTINUADO ASCENDENTEMENTE 2KM HLL - MTG

Figura 8. Mapa de la configuración del campo magnético reducido al polo y continuado ascendentemente a $2 \mathrm{~km}$. Límite del Graben de Villa de Reyes, SLP, en línea color blanco. 
entendimiento y facilidad de observación se marcaron en el mapa sólo algunos de los lineamientos relacionados con la tectónica del GVR (Figura 9).

En el mapa de $1 \mathrm{aDz}$ se muestra el lineamiento que existe entre el Graben de Bledos (hombro NW del GVR) y la Fosa de Melchor (Hombro SE del GVR) con una orientación general NW-SE, el lineamiento se observa interrumpido dentro del GVR por una estructura tipo fosa que tiene una orientación NE-SW.

En la configuración de la $1 \mathrm{aDz}$ también se observan la existencia de fosas dentro del GVR, sobresalen la ubicada en la porción sur y parte media del GVR. La zona de falla del SW de la ciudad de S.L.P. (porción superior del mapa) se distingue por su claro alineamiento en dirección NW-SE.

Otro de los algoritmos matemáticos utilizados en el estudio de la información aeromagnética, fue la señal analítica (Nabighian, 1972), un filtro para 2-D, y ampliado para 3-D (Roest et al., 1992), el cual ha sido utilizado ampliamente para localizar fronteras y profundidad de fuentes magnéticas (Hsu et al., 1996, 1998).

La señal analítica ( $S A$; Ecuación 3 ) se define como la raíz cuadrada de la suma de los cuadrados de las derivadas en las direcciones $X, Y, Z$.

$$
S A=\sqrt{ }\left(d x^{*} d e+d y^{*} d y+d z^{*} d z\right) \quad \text { (Ecuación 3) }
$$

En el mapa generado con la $S A$ (Figura 10), pudimos interpretar diferentes comportamientos de las señales magnéticas, el más importante fue la definición de al menos seis dominios aeromagnéticos (DAM) en la zona de estudio.

El DAM I ubicado en la porción SW está constituido por dos subdominios. El Ia se identifica al W del DAM y se interpreta como un escalón del hombro SW del GVR que se correlaciona con las Ignimbritas Panalillo Superior e Inferior, y ventanas de Ignimbrita Cantera, Latita Portezuelo y Riolita San Miguelito. El subdominio Ib localizado en la porción central, E y SE del DAM se ha interpretado como asociado a la posible presencia del Basalto Cabras en la zona. EL DAM en general tiene una morfología similar a una letra $U$, alargada en dirección NW-SE. La parte inferior de la letra U se correlaciona con el relleno aluvial del GVR.

El DAM II se localiza en la mayor parte del área y está representado por una amplia zona con orientación NW-SE, la cual contiene los DAM III, IV y V que se alinean con la misma dirección. El DAM II está dividido para su análisis en tres subdominios aeromagnéticos. El subdominio IIa se identifica en la porción NW mostrándose muy uniforme, apreciándose algunos rasgos NW-SE que pueden correlacionarse con zonas de falla con afloramientos puntuales de rocas máficas a lo largo de ellas. En los hombros se correlaciona con la Ignimbrita Cantera y coladas de la Riolita San Miguelito.

El subdominio IIb se correlaciona con el valle que conforma el GVR, sólo se ve interrumpido por el DAM III. Se conoce por información de pozos para agua, ya que gran parte de la fosa se encuentra ocupada irregularmente por domos de la Latita Portezuelo cuyo espesor puede alcanzar hasta los $500 \mathrm{~m}$. Hacia la porción SW el subdominio IIb se correlaciona con los depósitos de relleno más potentes. El subdominio IIc se localiza en la porción SE del DAM y se correlaciona principalmente con la Traquita Ojo Caliente cuyas coladas cubren casi toda la zona de este subdominio. En superficie existen remanentes del Basalto Cabras que también se asocian al subdominio IIc.

El DAM III se sitúa en la porción media central GVR y se le ha interpretado como la posible fuente de la Latita Portezuelo. En la mayor parte de la zona central y suroriental de este dominio, afloran ventanas de la Latita Portezuelo, la cual está sepultada dentro del GVR. Los pozos perforados para agua en esta zona han cortado espesores de esta roca hasta de $500 \mathrm{~m}$, encontrando en el fondo a la Ignimbrita Santa María. El DAM tiene una morfología de características amibiácicas.

EL DAM IV se le identifica en la porción media de la Sierra de San Miguelito en el hombro NW del GVR, tiene una forma alargada en dirección NE-SW y se le ha correlacionado con la fuente del domo El Zapote.

El DAM V se ubica en la porción NNW del área de estudio, tiene una forma trapezoide con eje mayor en dirección NW-SE y se le asocia con el Cerro Potosí, fuente de la Riolita San Miguelito. En la zona de este DAM aflora principalmente la Riolita San Miguelito que se ve cruzada por fallas normales en dirección NW-SE. Una de estas fallas principales que cruzan la Sierra de San Miguelito en esta zona, fue el conducto por donde se emplazaron puntualmente la Ignimbrita Panalillo Superior y el Basalto La Placa.

El DAM VI se localiza en la parte NE de la zona de estudio, no tiene una forma definida, pero se observa un lineamiento en dirección NW-SE en donde se ubican los domos El Potosí y El Fraile, los cuales forman las porciones más elevadas de la Sierra de San Miguelito. Hacia la zona del valle se ha interpretado como basamento de Latita Portezuelo, la cual alcanza profundidades de hasta 400m según perforaciones para localizar agua subterránea.

\subsection{Modelos de anomalías magnéticas}

En un intento por investigar la geometría y las propiedades magnéticas de los cuerpos fuentes asociados a las anomalías se realizaron dos modelos de las anomalías magnéticas en las secciones A-A' y B-B' utilizando el software $G M$ SYSTM (Marquardt, 1963) para invertir los cálculos (Figura 11). El GM-SYS ${ }^{T M}$ usa un implemento de este algoritmo para procesar datos magnéticos desarrollado por el Servicio Geológico Americano (USGS) en el programa de computo SAKI (Webring, 1985). Las anomalías son modeladas por medio de cuerpos poligonales con diferente magnetización. Una limitante mayor es la falta de información relacionada con la variación de las propiedades magnéticas con la profundidad. Un problema en la construcción de modelos cuantitativos es la heterogeneidad de las propiedades magnéticas observadas en los depósitos volcánicos. 


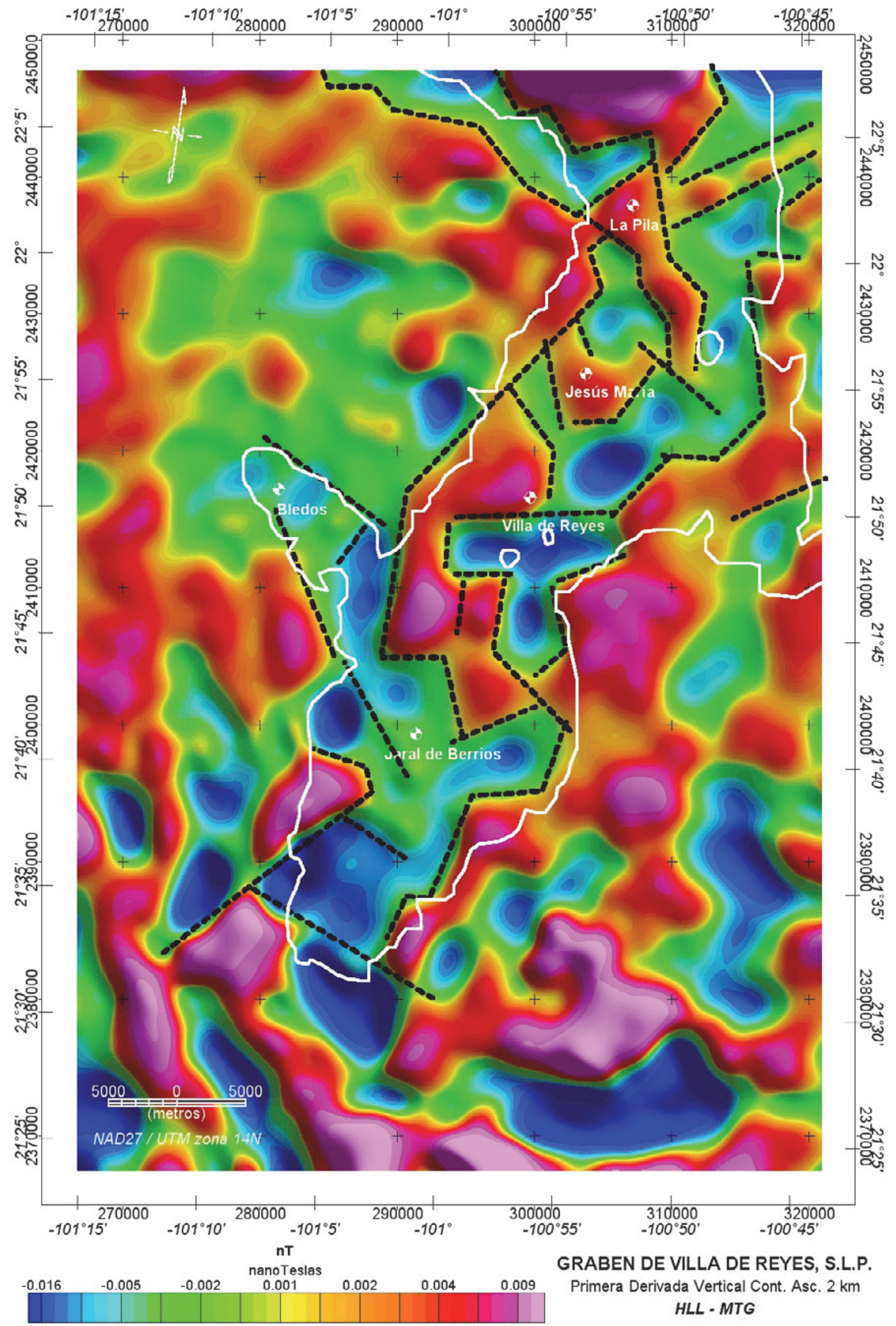

Figura 9. Mapa de la configuración de la primera derivada vertical del campo magnético reducido al polo y continuado ascendentemente a $2 \mathrm{~km}$, en donde se han marcado algunos de los lineamientos aeromagnéticos que existen en el Graben de Villa de Reyes, SLP. 


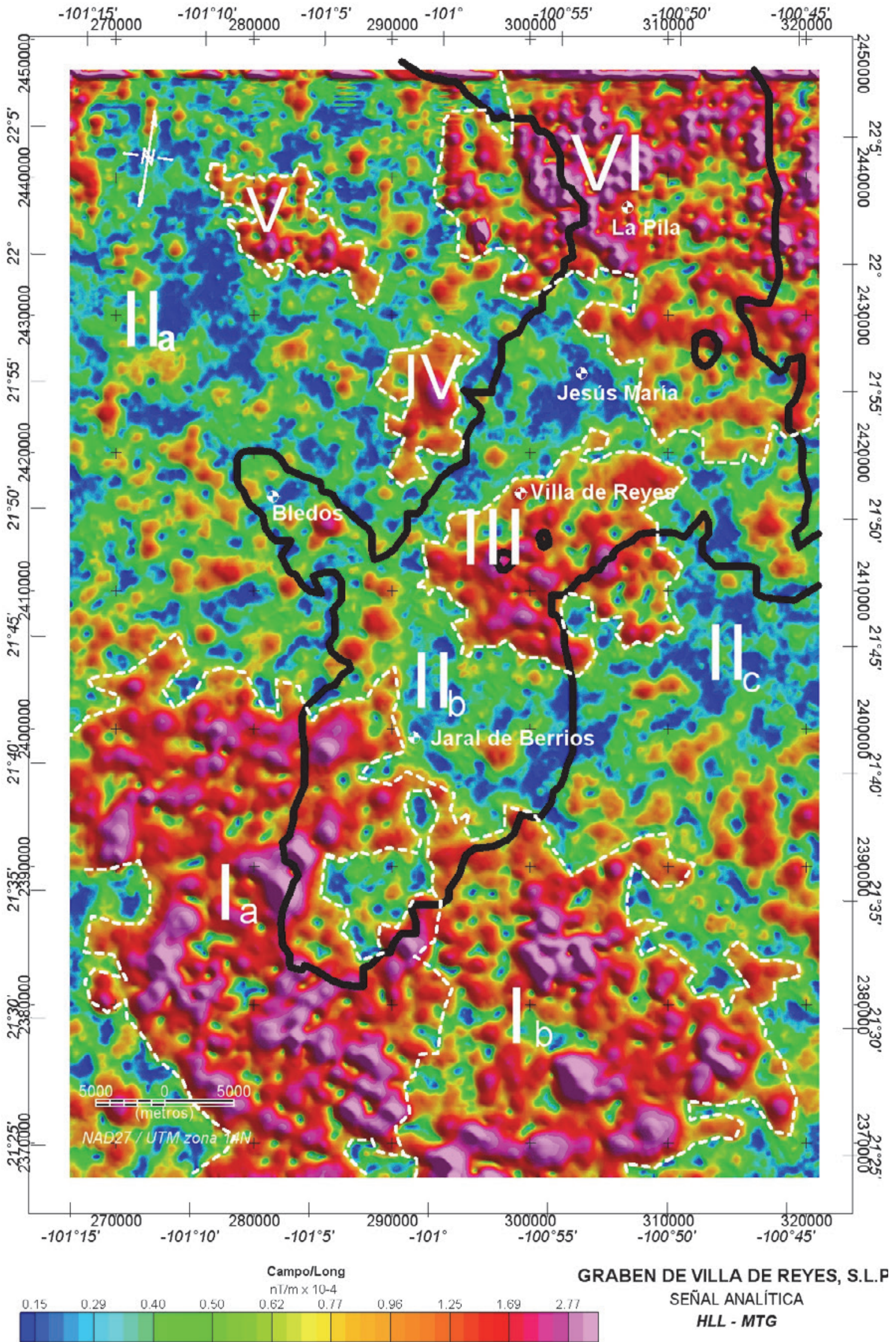

Figura 10. Dominios aeromagnéticos (DAM) interpretados a partir de la señal analítica del campo magnético reducido al polo. La línea blanca delimita a los DAM y la línea negra al Graben de Villa de Reyes, SLP. 

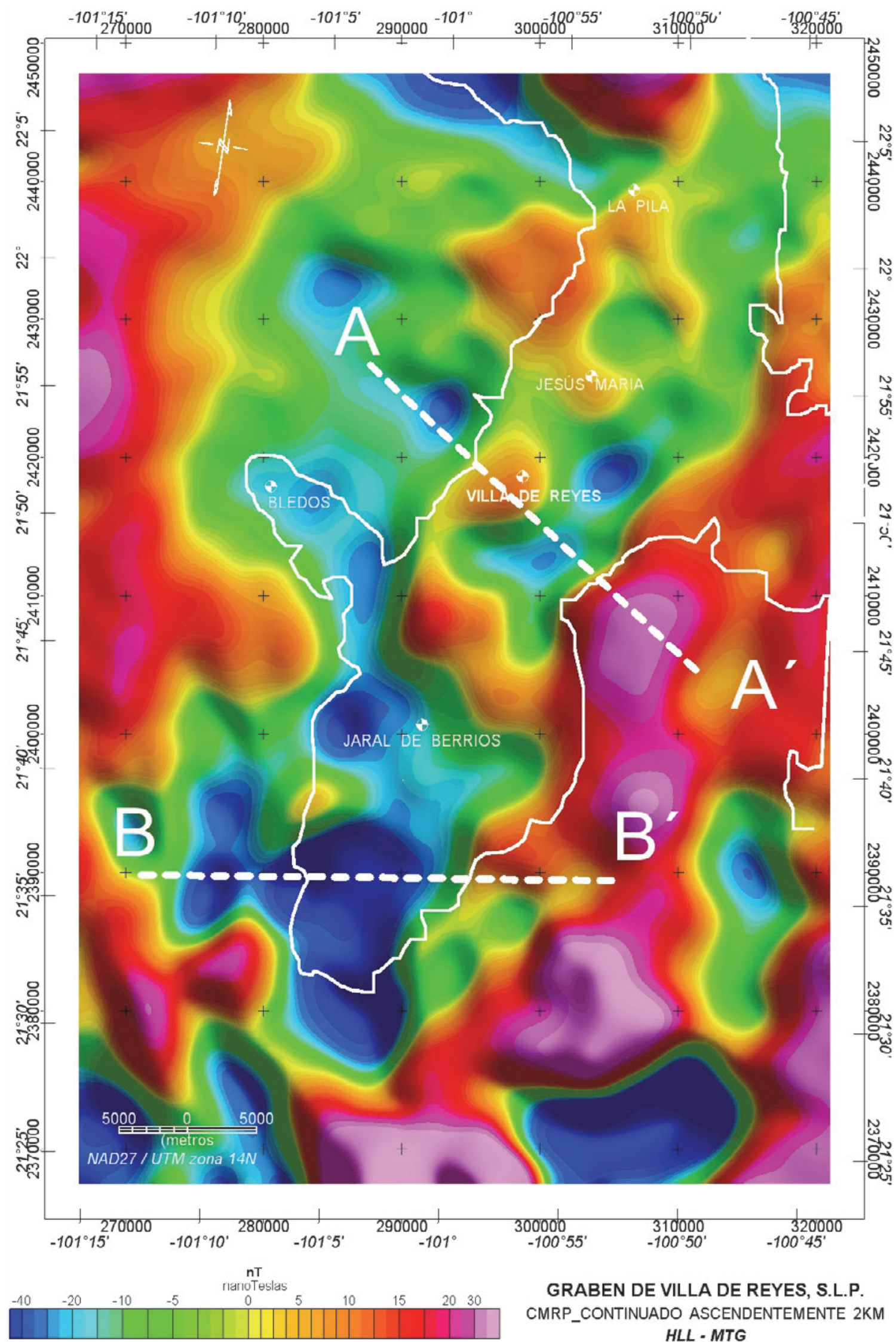

GRABEN DE VILLA DE REYES, S.L.P. CMRP_CONTINUADO ASCENDENTEMENTE 2KM $H L L$ - MTG

Figura 11. Ubicación de las secciones aeromagnéticas modeladas (A-A' y B-B') en el mapa de la configuración del campo magnético reducido al polo y continuado ascendentemente a $2 \mathrm{~km}$ del Graben de Villa de Reyes, SLP. 


\subsubsection{Sección $A-A^{\prime}$}

Se ubica hacia la porción central del Graben de Villa de Reyes. La sección magnética tiene una orientación NW$\mathrm{SE}$, viendo hacia el N, tendiendo a ser perpendicular a la dirección del GVR. El modelo magnético tiene un error menor al $10 \%$. Esta sección muestra en el hombro poniente del GVR en la Sierra de San Miguelito, un conjunto de fallas paralelas con orientación NW-SE, dispuestas en un patrón de fallas normales en domino que bascularon los bloques al NE. El modelo muestra también la presencia de dos zonas de falla en el basamento rocoso de la fosa del GVR. El hombro W del GVR se ve menos tectonizado que el hombro W. La zona de la fosa muestra un pilar hacia la porción centrooriente de la sección que delimita a dos valles.

\subsubsection{Sección $B-B^{\prime}$}

Esta se localiza hacia la porción SW del GVR, tiene una orientación W-E. La sección aeromagnética modelada muestra la clara correlación que existe entre zonas de debilidad (fallas y/o fracturas) y valores mínimos de magnetismo. Estas áreas se consideran como posibles zonas de recarga del acuífero de la zona. Obsérvese en el modelo la geología y la compleja tectónica que existe en esta porción del GVR. También se puede apreciar el gran espesor de relleno que existe al oriente del hombro SW del GVR.

\section{Conclusiones}

La magnetometría aérea refleja la existencia de rocas volcánicas e ígneas bajo la cubierta aluvial, las cuales se encuentran fracturadas y constituidas en su mayor parte por rocas volcánicas latíticas, asimismo se pudo observar las estructuras geológicas asociadas y que tienen relación con algunas de las zonas de recarga del acuífero del GVR.

Se correlacionaron algunas de las anomalías aeromagnéticas con las fuentes de rocas volcánicas que existen en el GVR. En general la magnetometría aérea permitió observar la existencia y ubicación de algunas de las fuentes de vulcanismo que existen en la Sierra de Santa María del Río (SSMR) y el la Sierra de San Miguelito, así como la presencia de algunos pilares existentes dentro del relleno aluvial del GVR.

Las zonas de falla y de recarga se muestra que están asociadas a bajos magnéticos. Las zonas más propicias para la ubicación de nuevas fuentes de abastecimiento son aquellas que se correlacionan con cruces de zonas de falla y/o fracturas asociadas con lineamientos aeromagnéticos, así como las zonas que presentan mayores espesores del relleno aluvial las cuales se ubican principalmente en la porción SW del GVR.

\section{Agradecimientos}

Esta investigación fue apoyada por los Fondos Mixtos FOMIX_SLP-2003-4722. Se agradece al Ing. David E. Torres-Gaytán, técnico del IPICYT, su apoyo en la elaboración de algunas de las figuras que se muestran en el trabajo.

\section{Referencias}

Aguillón-Robles, A., Aranda-Gómez J.J., Solorio-Munguía, J.G., 1994, Geología y tectónica de un conjunto de domos riolíticos del Oligoceno medio en el sur del estado de San Luis Potosí, México: Revista Mexicana de Ciencias Geológicas, 11, 29-42.

Aguillón-Robles, A., Tristán-González, M., Torres-Hernández, R., BustosGutiérrez, L.A., Carrizales-Aguilar, A., 2004, Carta GeológicoMinera Santa María del Río, F14-C14, 1:50000: Servicio Geológico Mexicano (SGM), 1 Mapa.

Aguirre-Hernández, M.A., 1992, Geología del subsuelo de las cuencas geohidrológicas del Valle de San Luis Potosí y de Villa de Reyes, en el estado de San Luis Potosí: Universidad Autónoma de San Luis Potosí, Instituto de Geología, Folleto Técnico No 116, 46 p.

Aranda-Gómez, J. J., Henry, C. D., Luhur, J. F., 2000, Evolución tectonomagmática post-paleocénica de la Sierra Madre Occidental y de la porción meridional de la provincia tectónica de Cuencas y Sierras, México: Boletín de la Sociedad Geológica Mexicana, 53 (1), 59-71.

Baranov V., Naudy, H., 1964, Numerical calculationof the formula of reduction to the magnetic pole: Geophysics, 29, 69-79

Bhattacharyya, B.K., 1971, An automatic method of compilation and mapping of high resolution aeromagnetic data: Geophyiscs, 36, 695-716

Blakely R. J., 1995, Potential Theory in Gravity \& Magnetic Applications: Cambridge University Press, 441p.

Comisión Federal de Electricidad (CFE), 1990, Características geohidrológicas en el área de la central termoeléctrica de San Luis Potosí: Comisión Federal de Electricidad. Subdirección de Construcción, Gerencia de Ingeniería Civil. Reporte Interno por Flores, S.A., Alcalá, Hernández, M., 114 p.

Consejo de Recursos Minerales (CRM), 1992, Monografía GeológicoMinera del Estado de San Luis Potosí: Pachuca, Hidalgo, México, Consejo de Recursos Minerales, $218 \mathrm{p}$.

Edwards, J.D., 1956, Studies of some early Tertiary red conglomerates of Central Mexico: U.S., Geological Survey, Professional Paper 264-H, 153-185.

Hanna, W.F., 1990, Some historical notes on early magnetic surveying in the U.S: Geological Survey, en Hanna, W.F. (ed.), Geologic Application of Modern Aeromagnetic Surveys, 63-73, U.S.: Geological Survey Bulletin 1924, Denver, CO.

Henderson, R.G., 1970, On the validity of the use of the upward continuation integral for total magnetic intensity data: Geophysics 35, 916-919.

Henderson, R.G., Zietz, I., 1949, The computational of second vertical derivates of geomagnetic fields: Geophysiscs 14, 508-516.

Henry, C.D., Aranda-Gómez, J.J., 1992, The real southern Basin and Range: Mid to late Cenozoic extension in Mexico: Geology, 20, 701-704.

Hsu, S.K., Sibuet, J.C., Shyu, C.T., 1996, High resolution detection of geological boundaries from potential-field anomalies: an enhanced analytic signal technique: Geophysics, 61, 373-386.

Hsu, S.K., Coppens D., Shyu, C.T., 1998, Depth to magnetic source using the generalized analytic signal: Geophysics 63, 1947-1957.

INEGI, 2009, Estadísticas censales a escalas geoelectorales, II Conteo de Población y Vivienda 2005: Instituto Nacional de Estadística y Geografía. Disco Compacto. 
Labarthe-Hernández, G., Tristán-González, M. y Aranda-Gómez, J.J., 1982, Revisión estratigráfica del Cenozoico de la parte central del Estado de San Luis Potosí: Universidad Autónoma de San Luis Potosí, Instituto de Geología y Metalurgia, Folleto Técnico 85, 208 p.

Labarthe-Hernández, G., Tristán-González, M., Martínez-Ruíz, V. J., 1983, Estudio geohidrológico de la Hoja Melchor, Estados de San Luis Potosí y Guanajuato: Universidad Autónoma de San Luis Potosí, Instituto de Geología y Metalurgia, Folleto Técnico 92, 109 p.

Labarthe-Hernández, G., Tristán-González, M., Aguillón-Robles, A., 1984, Cartografía geológica 1:50000, Hoja Salitrera, S.L.P: Universidad Autónoma de San Luis Potosí., Instituto de Geología y Metalurgia, Folleto Técnico 94, 85 p.

Labarthe-Hernández, G., De la Huerta Cobos, M.L., 1998, Geología del Semi-Graben de Bledos, San Luis Potosí, México, Escala 1:20000: Universidad Autónoma de San Luis Potosí, Instituto de Geología, Folleto Técnico 124, $33 \mathrm{p}$.

López-Loera, H., Urrutia-Fucugauchi, J. 1999, Spatial and temporal magnetic anomalies of Colima Volcano, Western Mexico: Geofísica Internacional, 38 (1), 3-16.

Marquardt, D. W., 1963, An algorithm for least-squares estimation of nonlinear parameters: Journal of the Society for Industrial and Applied Mathematics, 11, 431-441.

Martínez-Ruiz, V. J., Cuellar-González, G., 1979, Correlación de superficie y subsuelo de la Cuenca Geohidrológica de San Luis Potosí. S.L.P.: Universidad Autónoma de San Luis Potosí, Instituto de Geología y Metalurgia, Folleto Técnico 65, 25 p.

Nabighian, M.N., 1972, The analytic signal of two-dimensional magnetic bodies with poligonal cross section: its properties and use for automated anomaly interpretation, Geophysics, 37, 507-517

Nieto-Samaniego, A.F., Alaniz-Álvarez, S.A., Labarthe-Hernández, G. 1997, La deformación cenozoica poslaramídica en la parte meridional de la Mesa Central, México: Revista Mexicana de Ciencias Geológicas, 14, 13-25.

Ramos-Leal J.A., López-Loera, H., Martínez-Ruiz V.J., Aranda-Gómez, J.J., 2007, Sucesión de eventos y geometría de la parte central del acuífero de Villa de Reyes (San Luis Potosí, México) inferida a partir de datos geoeléctricos. Revista Mexicana de Ciencias Geológicas, 24 (1), 31-46.

Robinson, E.S., Coruh, C., 1988, Basic Exploration Geophysics: Wiley \& Sons, $562 \mathrm{p}$.
Rodríguez-Ríos, R., 2003, Estudio Geoquímico preliminar del vulcanismo bimodal en el Semigraben de Bledos, Campo Volcánico de San Luis Potosí, en XIII Congreso Nacional de Geoquímica, Actas INAGEQ, 9 (1), 143.

Roest, W. R., Verhoef, J., Pilkington, M., 1992, Magentic interpretation using the 3-D analytic signal, Geophysics, 57, 116-125.

Stewart, J.H., 1998, Regional characteristics, tilt domains, and extensional history of the late Cenozoic Basin and Range province, western North America: Geological Society of America Special Paper 323, 47-74.

Telford W.M., Geldart, L.P., Sheriff, R.E., 1990, Applied Geophysics: Cambridge University Press, $770 \mathrm{p}$.

Torres-Aguilera, J.M. 2005, Caracterización petrográfica y geoquímica del vulcanismo bimodal en el Semigraben de Bledos, en el Campo Volcánico de San Luis Potosí: Universidad Autónoma de San Luis Potosí, Facultad de Ingeniería, Tesis de Maestría, $159 \mathrm{p}$.

Torres-Aguilera, J. M., Rodríguez-Ríos, R., 2005, Hipótesis preliminares sobre el origen del volcanismo bimodal en el Campo Volcánico de San Luis Potosí, en XV Congreso Nacional de Geoquímica, Actas INAGEQ, 11 (1), p. 107.

Tristán-González, M., 1986, Estratigrafía y tectónica del graben de Villa de Reyes, en los estados de San Luis Potosí y Guanajuato, México: Instituto de Geología y Metalurgia, Universidad Autónoma de San Luis Potosí, Folleto Técnico 107, 91 p.

Tristán-González, M., Aguillón-Robles, A., Barboza-Gudiño, J. R., TorresHernández, J.R., Herve, B., López-Doncel, R., Rodríguez-Ríos, R., Labarthe-Hernández, G., 2009, Geocronología y distribución espacial del vulcanismo en el Campo Volcánico de San Luis Potosí: Boletín de la Sociedad Geológica Mexicana, 61 (3), 287-303.

Webering, M., 1985, SAKI: A Fortran program for generalized linear inversión of gravity and magnetic profiles: US Geological Survey, Open File Report 85-122, 29p.

Manuscrito recibido: Mayo 17, 2010.

Manuscrito corregido recibido: Octubre 7, 2010.

Manuscrito aceptado: Enero 19, 2011. 